\title{
Dinâmica populacional da matrinxã Brycon amazonicus (Characidae) na Amazônia Central
}

\author{
Leocy C. dos Santos Filho ${ }^{1} \&$ Vandick da S. Batista ${ }^{2,3}$
}

\begin{abstract}
${ }^{1}$ Rua Santa Helena 547, Betania, 69073-070 Manaus, Amazonas, Brasil. E-mail: leocy_cutrim@yahoo.com.br
2 Universidade Federal de Alagoas, Instituto de Ciências Biológicas e da Saúde/ICBS, Universidade Federal de Alagoas. Praça Afrânio Jorge, Prado, 57010-020 Maceió, Alagoas, Brasil. E-mail: vbatista@pq.cnpq.br

${ }^{3}$ Corresponding author.
\end{abstract}

\begin{abstract}
Population dynamics of matrinxã Brycon amazonicus (Characidae) in Central Amazon. The matrinxã, Brycon amazonicus (Spix \& Agassiz, 1829) is one of the most important fishery resources of the Amazonas state. Its population dynamics in Central Amazon was analyzed based on total landing and biometry data registered in the main landing port of Manaus, between 1994 and 2002. Growth and mortality rates were estimated separately for the rivers Purus, Madeira and Solimões. Differences in size structure and growth curves suggest that different population units exist among these rivers, requiring individualized evaluation and fisheries management strategies. The analysis of the yield per recruit does not indicate overexploitation. However, the highest relative exploitation rate was observed in the Madeira river. The suggested management strategies are related to restrictions to the fishery in the main fishing grounds during the migratory dispersal period, instead of restrictions during reproductive periods.
\end{abstract}

KEY WORDS. Fisheries; growth; mortality; stock.

Caraciformes é o grupo de peixes de maior riqueza específica da Região Amazônica (Ferreira et al. 1996) e é o grupo cujas espécies fornece a maior parte de biomassa de pescado disponível para consumo na Amazônia Central (BATISTA et al. 2004), sofrendo elevado esforço de pesca (Batista \& Petrere 2003). As espécies que mais se destacam nos desembarques são o jaraqui escama fina, Semaprochilodus taeniurus (Valenciennes, 1821), o jaraqui escama grossa Semaprochilodus insignis (Jardine \& Schomburgk, 1841), o curimatã Prochilodus nigricans Spix \& Agassiz,1829, o pacu - vários Myleinae, principalmente Mylossoma duriventre (Cuvier, 1818), matrinxã Brycon amazonicus (Spix \& Agassiz, 1829) - e tambaqui Colossoma macropomum (Cuvier, 1816). Estes caraciformes vivem sazonalmente em áreas alagadas e utilizam o canal do rio como via migratória, sendo capturados nos dois ambientes por pescadores de subsistência, comerciais e esportivos.

Brycon amazonicus é conhecida com o nome vulgar de matrinxã na maior parte da Amazônia Central (SANTos et al. 2006, Lima 2003), sendo também chamada na bacia do rio Madeira de "jatuarana" (Goulding 1979, 1980). Tem distribuição ao longo do Rio Solimões-Amazonas e tributários, na bacia do rio Orinoco e no rio Essequibo na Venezuela (Lima 2003). A espécie é migradora, onívora, se reproduz com desova total no início da enchente e seus principais predadores são os botos, Inia geoffrensis (Blainville, 1817) e Sotalia fluviatilis (Gervais \& Deville, 1853) (Sмith 1979, Goulding 1979, 1980, Santos et al. 2006). Sua importância na pesca da Amazônia Central é gran- de em termos comerciais (Petrere 1978, Saint-Paul \& Bayley 1979, Smith 1979, Batista \& Petrere 2003, Batista et al. 2007) e na pesca de subsistência (Sмith 1979, Batista et al. 2000), assim como ocorre no médio Amazonas (Ferreira et al. 1996), sendo que atualmente também é a segunda espécie mais produzidas em pisciculturas no Amazonas (IвAма 2007).

Estatísticas de desembarque realizadas no período de 1979-1987, em nove municípios do Estado do Amazonas, colocaram a matrinxã como o quinto pescado mais comercializado, com um total de 11.446 toneladas (Sudepe 1988). Destacou-se ainda entre os mais desembarcados em Manaus no período de 1994 e 1996 (Batista \& Petrere 2003). Ibama (2003, 2004a, b, 2005, 2007) também aponta a matrinxã entre o quarto e sétimo lugar dentre os pescados mais comercializadas no Amazonas entre 2001 e 2005, apresentando um período de safra entre os meses de abril a junho, sendo capturada antes da safra de vazante/verão das espécies curimatá, pacus e sardinhas.

Um dos objetivos das pesquisas sobre recursos pesqueiros economicamente importantes é embasar tecnicamente o estabelecimento de regras para sua explotação racional, através do conhecimento da dinâmica das populações componentes (Hilborn \& Walters 1992, King 1995). Entretanto, as atividade de conservação ou uso da espécie, tais como limitações de tamanho de captura ou de defeso, tem sido efetuadas a partir de referenciais técnico científicos que justifiquem tais ações. Neste contexto, a determinação de parâmetros de crescimento, taxas de mortalidade natural e por pesca, assim como a aplica- 
ção de modelos quantitativos (GulLAND 1983), podem permitir a predição de efeitos das medidas propostas para o manejo (Hilborn \& Walters 1992, Sparre \& Venema 1997), como já indicado para espécies amazônicas, tais como o tambaqui (IsAac \& Ruffino 1996) ou a piramutaba (BARThem \& Petrere 1996). Tais parâmetros e modelos também são amplamente utilizados para a estimativa de pontos referenciais técnicos por meta e limite (CADDY \& MAHON 1996), necessários para referenciar o diagnóstico do estado dos recursos em relação às metas do manejo.

Estudos de crescimento utilizando dados de freqüência de comprimento são de baixo custo em relação a coleta de estruturas rígidas ou por meio de marcação e recaptura, sendo particularmente adequadas quando os recursos humanos e financeiros para avaliação de estoques são muito limitados (SPARRE \& Venema 1997).

A matrinxã B. amazonicus, já foi alvo de pesquisas focadas na determinação de idade e crescimento na década de 1980 (BARTHEM \& FABRé 2004) e na avaliação de estoques com dados de 1994-1996 (Batista 2001). Entretanto, em nenhum estudo houve estimativas de parâmetros populacionais por calha que permitam avaliar a existência de diferentes populações, as quais demandariam uma gestão pesqueira diferenciada.

Neste contexto, o presente trabalho teve como objetivo determinar as características populacionais de B. amazonicus, para os sub-sistemas: rio Purus, rio Madeira, Médio rio Solimões e Baixo rio Solimões, como base para pesquisas de identificação de potenciais unidades populacionais, e sugerindo ações de manejo para o uso sustentável do recurso.

\section{MATERIAL E MÉTODOS}

As informações de desembarque foram obtidas a partir do banco de dados do Programa Integrado de Recursos Aquáticos e da Várzea/PYRÁ da Universidade Federal do Amazonas, por meio de entrevistas sobre a captura, esforço de pesca e local de pesca, sendo efetuadas por coletores contratados nos principais portos de desembarque de pescado de Manaus (mercados da Panair e do Adolpho Lisboa), no período entre janeiro de 1998 e dezembro de 1999.

As unidades espaciais foram estabelecidas considerando meso-bacias hidrográficas que mais contribuíram para a produção pesqueira da espécie no período. Assim, apenas os registros de dados de matrinxã provenientes das calhas dos rios Purus, Solimões e Madeira foram considerados (Fig. 1). Para o rio Solimões, considerando sua dimensão e características hidrográficas, houve a separação em dois sub-sistemas, o Médio Solimões (desde a desembocadura do rio Juruá e do Lago Coari) e o Baixo Solimões (entre as desembocaduras do Lago Mamiá e do rio Negro). Todas as pescarias registradas neste estudo ocorreram no Estado do Amazonas.

Durante o desembarque do pescado foram efetuadas medidas de comprimento furcal (CF) em centímetros, de exemplares selecionados aleatoriamente, registrando-se o local de origem do pescado. Os coletores foram orientados a obter um

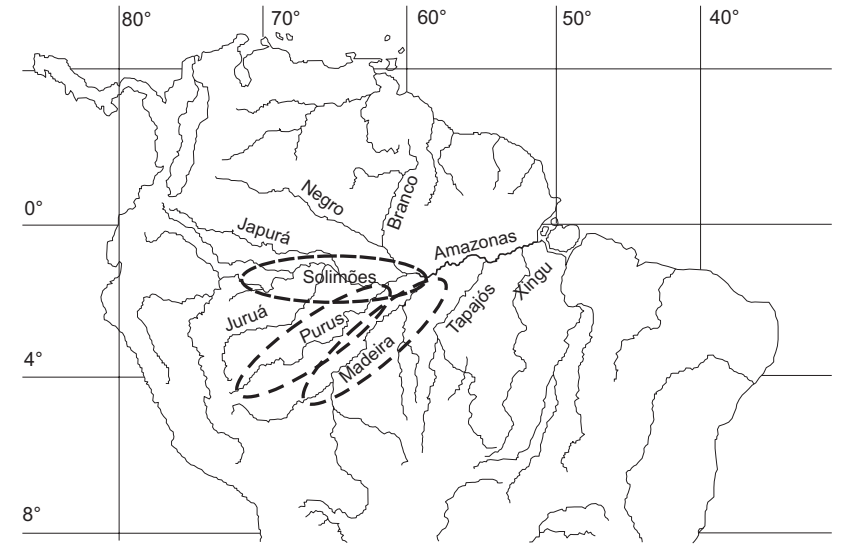

Figura 1. Sub-sistemas da Amazônia Central explotados pela frota pesqueira de Manaus que capturou os exemplares de matrinxã analisados neste trabalho.

mínimo de 30 medidas por noite, durante 10 dias por mês, buscando totalizar pelo menos 300 medidas por mês no total. O número de espécimes amostrado por embarcação foi definido a cada noite, de forma a obter dispersão espacial e temporal das amostras, visando representar a variabilidade da espécie.

As distribuições de freqüências de comprimento furcal de B. amazonicus foram analisadas em classes de $2 \mathrm{~cm}$ de comprimento, de forma a obter cerca de 15 a 20 classes recomendadas para as análises (SOKAL \& RoHLF 1995). Foram obtidos o comprimento mínimo (Lmin), comprimento máximo (Lmax), comprimento médio (L), desvio padrão (DP), variância e percentagem do coeficiente de variação da amostra $(\% \mathrm{CV})$ para as calhas dos rios Purus, Madeira e Solimões-Amazonas.

As análises foram efetuadas com a rotina ELEFAN (Eletronic Length Frequency ANalysis), utilizada pela FAO no programa FiSAT (Fish Stock Assessment Tools), o qual estima os parâmetros $\mathrm{L}_{\infty}$ e k da CCVB a partir de dados de distribuição de freqüência de comprimento de classes de comprimento dispostas seqüencialmente e as progressões das modas servem para inferir sobre o crescimento (Longhurst \& PAuly 1987). Para ajuste de modelo é assumida a equação generalizada de crescimento de von Bertalanffy como adequada, a qual contêm os parâmetros $\mathrm{C}$ e WP, cuja oscilação é freqüentemente relacionada à temperatura média do habitat de uma espécie (Pauly \& Morgan 1987).

Para calcular os parâmetros de crescimento, foi usada primeiramente a rotina de busca de valores do coeficiente de crescimento (k) do programa FISAT (GAYANILO \& PAUly 1997), a qual estima a aderência da curva de crescimento de von Bertallanfy (CCVB) aos dados de freqüência de comprimento entre 1998 e 1999. O comprimento máximo foi estimado também para estabelecer pontos referenciais. Estabelecido os pontos de partida para cálculo dos referidos parâmetros, foi efetuada a análise da superfície de resposta do ELEFAN, inserida no programa FISAT, para obter os valores de k e do comprimento assintótico $\left(\mathrm{L}_{\infty}\right)$. 
O WP ("ponto de inverno" ou de redução do crescimento) foi calculado com a fórmula: $\mathrm{WP}=\mathrm{ndv} / 365$, sendo ndv o número de dias até a vazante (período de menor crescimento entre julho-agosto).

A estimativa do parâmetro C (oscilação de crescimento) foi estabelecida a partir da variação anual da temperatura em torno de $5^{\circ} \mathrm{C}$ entre os períodos de enchente-cheia e vazanteseca (Junk \& KRAmbeck 2000), o que indica o valor de 5 para C.

A comparação da performance de crescimento foi efetuada por meio da estimativa do índice de performance do crescimento $\left(\varnothing^{\prime}\right)$ (Gayanilo \& Pauly 1997). O coeficiente de variação $(\mathrm{CV})$ de $\varnothing^{\prime}$ para vários estoques de uma mesma espécie não deve exceder $5 \%$.

O valor de $\varnothing^{\prime}$ foi estimado conforme a expressão (PAULY $\&$ Munro 1984): $\varnothing^{\prime}=\log _{10}(\mathrm{k})+2 \log _{10}\left(\mathrm{~L}_{\infty}\right)$

A estimativa de $t_{0}$ foi obtida pela relação empírica (PAULY 1983): $\log _{10}\left(-t_{0}=-0,3922-0,2752 \log _{10} L_{\infty}-1,038 \log _{10} \mathrm{~K}\right.$.

O modelo de von BertalanfFy (1938) foi utilizado para o ajuste da curva de crescimento para os dados de comprimento. A equação utilizada é aquela modificada por GAYANILO \& Pauly (1997).

A mortalidade total foi estimada pela equação de BeVerton \& Holt (1956). A mortalidade natural (M) foi obtida pela aplicação dos modelo de PAULY (1983) para peixes de cardume $\left(\mathrm{M}^{\prime}\right): \mathrm{M}^{\prime}=0,8 \mathrm{e}^{\left(-0,0152-0,279 \ln \left(\mathrm{L}_{\infty}\right)+0,6543 \operatorname{lm}(\mathrm{k})+0,463 \ln (\mathrm{T})\right)}$, onde: $\mathrm{T}=$ temperatura média anual na superfície da água. A mortalidade por pesca (F) foi estimada como a diferença entre a taxa instantânea de mortalidade total (Z) e a taxa instantânea de mortalidade natural para peixes de cardume $\left(\mathrm{M}^{\prime}\right): \mathrm{F}=\mathrm{Z}-\mathrm{M}^{\prime}$

A idade de recrutamento ( $\mathrm{Tr})$ foi estimada a partir do comprimento médio de recrutamento (Lr), que indica o tamanho no qual 50\% dos indivíduos jovens (<30 cm CT, segundo Zaniboni \& Resende 1988) que ingressam na área de pesca estão passíveis de captura pela redinha (rede de cerco). As proporções foram ajustadas ao modelo logístico na forma sugerida por KING (1995).

O rendimento por recruta foi estimado pelo modelo de Beverton \& Holt (1957). O peso assintótico do corpo foi obtido por meio da conversão dos comprimentos utilizando a equação: Peso total $=0,011+\mathrm{CF}^{3,143}\left(\mathrm{r}^{2}=0,94, \mathrm{n}=232, \mathrm{p}<\right.$ $0,05)$, obtida a partir de coleta de comprimentos e pesos de pescados no desembarque e em feiras de Manaus, Amazonas.

Para a análise do rendimento por recruta foram traçadas várias curvas para diferentes comprimentos de recrutamento pesqueiro (Cr), sendo estimados os valores do ponto referencial por meta da mortalidade por pesca ao nível de 10\% da razão inicial de incremento do rendimento por recruta $\left(\mathrm{F}_{0,1}\right)$ (PAULY 1984) para cada sub-sistema, assumindo o tamanho de recrutamento de $30 \mathrm{~cm} \mathrm{CF}$.

As medidas diárias do nível dos rios foram obtidas para todo o período com dados cedidos pela Agência Nacional de Águas (ANA) por meio de consulta em www.hidroweb.ana. gov.br para as seguintes estações: Borba $\left(4^{\circ} 23^{\prime} \mathrm{S}, 59^{\circ} 36^{\prime} \mathrm{W}\right)$, Beruri $\left(2^{\circ} 00^{\prime} \mathrm{S}, 61^{\circ} 32^{\prime} \mathrm{W}\right)$, Manacapuru $\left(3^{\circ} 19^{\prime} \mathrm{S}, 60^{\circ} 35^{\prime} \mathrm{W}\right)$, São Paulo de Olivença $\left(3^{\circ} 28^{\prime} \mathrm{S}, 68^{\circ} 45^{\prime} \mathrm{W}\right)$, Humaitá $\left(7^{\circ} 30^{\prime} \mathrm{S}, 63^{\circ} 01^{\prime} \mathrm{W}\right)$, Manicoré $\left(5^{\circ} 49^{\prime} \mathrm{S}, 61^{\circ} 18^{\prime} \mathrm{W}\right)$ e Canutama $\left(6^{\circ} 32^{\prime} \mathrm{S}, 64^{\circ} 19^{\prime} \mathrm{W}\right)$. Para o porto de Manaus $\left(3^{\circ} 08^{\prime} \mathrm{S}, 60^{\circ} 00^{\prime} \mathrm{W}\right)$ foram usados os dados cedidos pela Companhia de Pesquisa de Recursos Minerais, Superintendência Regional do Amazonas.

\section{RESULTADOS}

A estrutura de tamanhos apresentou tendência a manterse similar entre os sub-sistemas (Fig. 2), com maior predominância de exemplares entre 29 e $37 \mathrm{~cm}$ de comprimento furcal (CF). Pode-se ainda identificar a ocorrência de alguns grupos modais, com destaque para classes vizinhas aos $29 \mathrm{~cm}$ e $33 \mathrm{~cm}$.

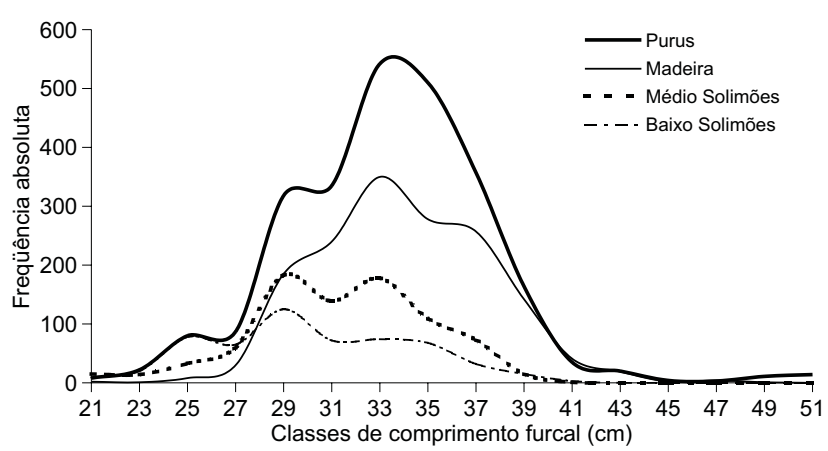

Figura 2. Comparação da distribuição de freqüência de comprimento da matrinxã entre 1998-1999 nos sub-sistemas estudados.

A matrinxã do rio Purus apresentou o maior percentual do coeficiente de variação e o menor foi do Médio Solimões (Tab. I). Comparando o comprimento furcal médio (L) dos quatro sub-sistemas estudados não encontramos diferença significativa (ANOVA p > 0,05).

Os parâmetros de crescimento para B. amazonicus, nos quatro sub-sistemas estudados foram estimados (Tab. II), gerando curvas de crescimento de von Bertalanffy (CCVB) apresentadas na figuras 3-6.

As curvas de crescimento de von Bertalanffy (CCVB) sazonais apresentaram maior similaridade entre os sub-sistemas do Solimões (baixo e médio), que diferem dos demais (Purus e Madeira), sendo indício de existirem curvas distintas para os sub-sistemas Purus, Madeira e Solimões (Fig. 7).

Os parâmetros utilizados para o calculo da mortalidade total e natural foram indicados nas tabelas I e II, sendo que o Tr foi de $20 \mathrm{~cm}$, o Tc observado foi de $25 \mathrm{~cm}$ para Purus, Baixo e Médio Solimões e de $27 \mathrm{~cm}$ para o Madeira, e a temperatura média utilizada foi de $28^{\circ} \mathrm{C}$ (Junk \& Krambeck 2000).

As estimativas de mortalidade (Tab. III) indicaram que dos sub-sistemas estudados, a matrinxã do rio Madeira apresentou maior mortalidade total (Z), enquanto que o do Mé- 
Tabela I. Estatística descritiva para a matrinxã dos rios Purus, Madeira, e Solimões (baixo e médio) nos anos 1998-1999. ( $\mathrm{L}_{\mathrm{min}}$ ) menor pescado medido, $\left(\mathrm{L}_{\text {máx }}\right)$ maior, $(\mathrm{L})$ tamanho médio registrado.

\begin{tabular}{|c|c|c|c|c|}
\hline Item & Purus & Madeira & Baixo Solimões & Médio Solimões \\
\hline Número & 2510 & 1560 & 561 & 820 \\
\hline $\mathrm{L}_{\min }$ & 20,0 & 20,0 & 16,0 & 20,0 \\
\hline $\mathrm{L}_{\text {máx }}$ & 52,0 & 50,0 & 38,0 & 40,0 \\
\hline $\mathrm{L}$ & 33,4 & 34,0 & 30,1 & 31,3 \\
\hline Desvio Padrão & 4,2 & 3,3 & 2,9 & 2,8 \\
\hline Coeficiente de Variação (\%) & 12,0 & 9,7 & 9,7 & 8,9 \\
\hline
\end{tabular}

Tabela II. Estimativas de parâmetros populacionais para a matrinxã nos sub-sistemas Purus, Madeira, Baixo Solimões e Médio Solimões. $\left(\mathrm{L}_{\infty}\right)$ indica o comprimento assintótico, $(\mathrm{k})$ constante de crescimento, (to) a idade teórica de tamanho 0, (C) a intensidade das oscilações senoidais, (WP) momento de redução do crescimento, $(\varnothing)$ índice de performance do crescimento.

\begin{tabular}{lcccccc}
\hline Sub-sistema & $\mathrm{L}_{\infty}(\mathrm{cm})$ & $\mathrm{k}($ ano $)$ & to (ano) & $\mathrm{C}$ & $\mathrm{WP}$ & $\varnothing$ \\
\hline Purus & 51,00 & 0,57 & $-0,54$ & 0,29 & 0,46 & 3,17 \\
Madeira & 51,10 & 0,72 & $-0,64$ & 0,29 & 0,46 & 3,27 \\
Baixo Solimões & 43,20 & 0,54 & $-0,49$ & 0,29 & 0,66 & 3,00 \\
Médio Solimões & 46,90 & 0,43 & $-0,40$ & 0,29 & 0,66 & 2,98 \\
\hline
\end{tabular}

Tabela III. Estimativas de mortalidade total, natural e por pesca, para os sub-sistemas rio Purus, rio Madeira, baixo rio Solimões e médio rio Solimões.

\begin{tabular}{lcccc}
\hline Sub-Sistema & $\begin{array}{c}\text { Mortalidade Total } \\
(\mathrm{Z} \mathrm{ano}-1)\end{array}$ & $\begin{array}{c}\text { Mortalidade Natural } \\
\left(\mathrm{M} \mathrm{ano}^{-1}\right)\end{array}$ & $\begin{array}{c}\text { Mortalidade por Pesca } \\
\left.(\mathrm{F} \mathrm{ano})^{-1}\right)\end{array}$ & $\begin{array}{c}\text { Taxa de Explotação } \\
(\mathrm{E} \mathrm{ano})\end{array}$ \\
\hline Purus & 1,20 & 1,06 & 0,14 & 0,12 \\
Madeira & 1,76 & 1,24 & 0,52 & 0,30 \\
Baixo Solimões & 1,39 & 1,07 & 0,32 & 0,23 \\
Médio Solimões & 1,06 & 0,90 & 0,16 & 0,15 \\
\hline
\end{tabular}

dio Solimões apresentou a menor. Em relação às estimativas de mortalidade natural (M), a matrinxã do Madeira apresentou o maior valor, e o menor foi apresentado pela matrinxã do Médio Solimões. Já as estimativas de mortalidade por pesca (F) variaram entre o maior valor de 0,52 para o Madeira, e o menor valor de 0,14 para o rio Purus.

Comparando-se os valores estimados para taxa de explotação pesqueira para os sub-sistemas estudados, foi observado que os sub-sistemas Madeira e Baixo Solimões apresentaram uma taxa de explotação mais elevada, seguidos por Médio Solimões e por último pelo Purus com a menor taxa de explotação.

Para efeito de comparação entre os sub-sistemas estudados, foi avaliado o rendimento por recruta em função de F e para diferentes tamanhos de recrutamento pesqueiro, utilizando os parâmetros calculados para cada sub-sistema. Em todos os sub-sistemas estudados, os valores de $\mathrm{F}_{0,1}$ foram sempre superiores ao estimado para os atuais níveis de explotação da matrinxã, indicando sub-explotação em todos os sub-sistemas avaliados (Fig. 8). Considerando que o tamanho de recrutamento no período esteve em torno de $30 \mathrm{~cm} \mathrm{CF}$, os valores mais próximos do nível atual de $\mathrm{F}$ ao $\mathrm{F}_{0,1}$ foram registrados no Madeira $\left(\mathrm{F}_{\text {atual }}=0,52 ; \mathrm{F}_{0,1}=1,5\right)$, seguido pelo Baixo Solimões $\left(\mathrm{F}_{\text {atual }}=0,32 ; \mathrm{F}_{0,1}=1,6\right)$, Purus $\left(\mathrm{F}_{\text {atual }}=0,14 ; \mathrm{F}_{0,1}=\right.$ $1,0)$ e Médio Solimões $\left(F_{\text {atual }}=0,16 ; F_{0,1}=1,2\right)$,.

\section{DISCUSSÃO}

Na amostragem de capturas comerciais de peixes, Sparre \& Venema (1997) destacam a importância de se conhecer aspectos das pescarias, tais como: áreas de distribuição da espécie, tipos de embarcação que a explotam e os principais apetrechos utilizados. Para a matrinxã, Goulding (1979), Batista (2001), Lima (2003) e BARTHEM \& FABRÉ (2004) apresentaram as informações mais relevantes do conhecimento existente, abordando aspectos de pesca, biologia reprodutiva, parâmetros de crescimento por meio de estruturas rígidas, biologia pesqueira e taxonomia.

Trabalhos de crescimento de peixes na região têm sido efetuados tanto com estruturas rígidas como com distribuições de freqüência de comprimento. Os trabalhos com estruturas rígidas na região têm comprovado a existência de marcas peri- 

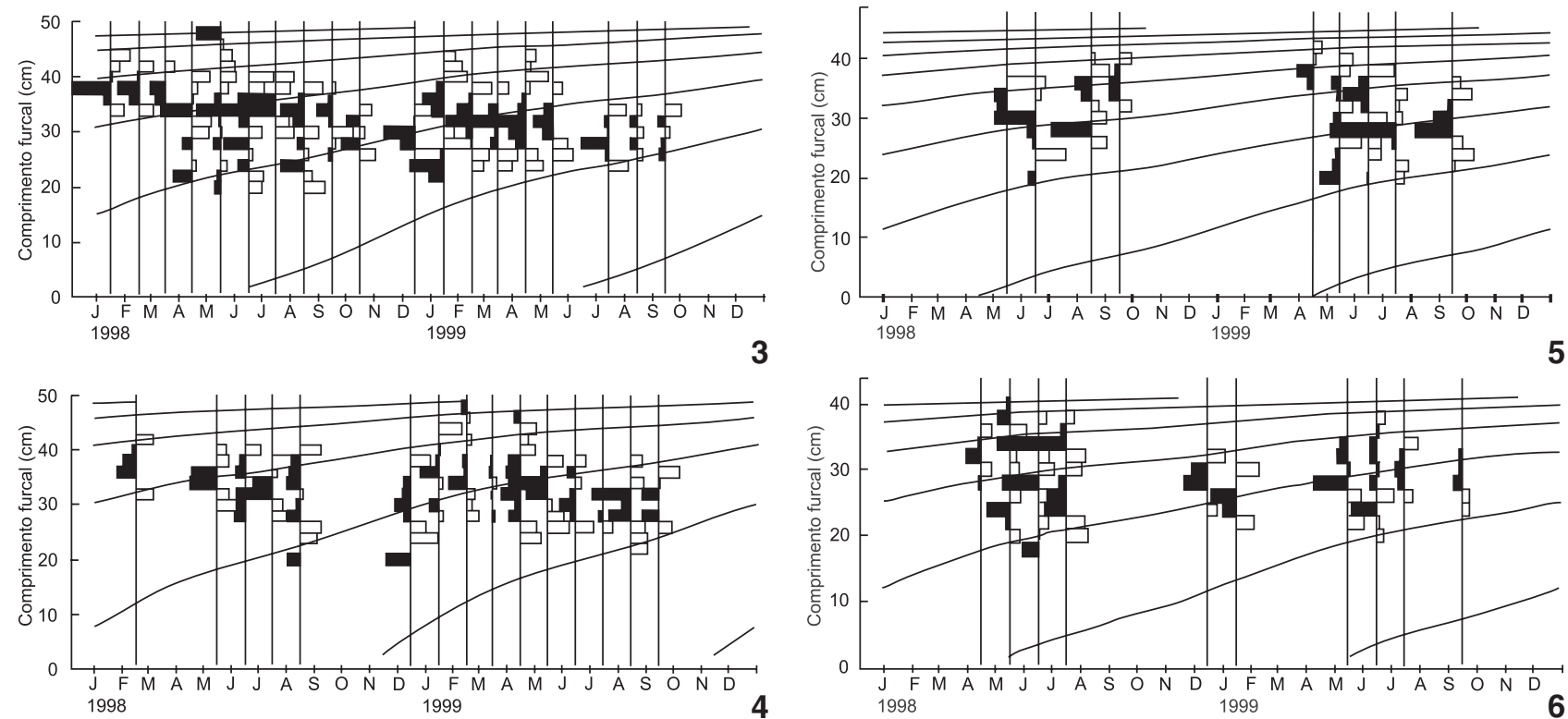

Figura 3-6. Distribuição ajustada de freqüências de comprimento do sub-sistema Purus (3), Madeira (4), Baixo Solimões (5) e Médio Solimões (6) com dados de 1998 e 1999 e linha de ajuste da CCVB de cada sub-sistema. As barras negras representam picos modais positivos e as brancas indicam depressões nas distribuições de freqüência mensais.

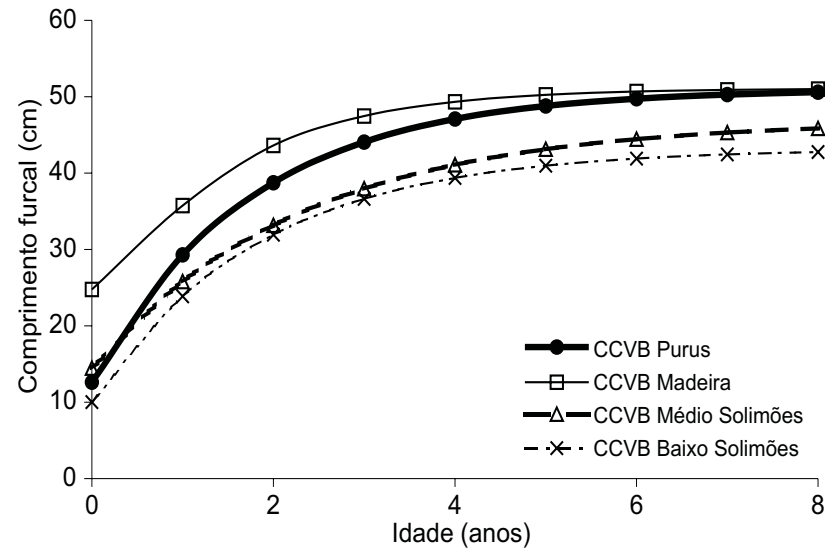

Figura 7. Curvas de crescimento de von Bertallanfy nos quatro sub sistemas estudados.

ódicas bem definidas em diversas estruturas calcificadas em peixes, como escamas, otólitos, espinhos e vértebras (Fabré \& SaINTPaul 1998, Santos-Filho \& Batista 2005), sendo porém estudos que exigem recursos humanos, materiais e infra-estrutura que não têm permitido uso continuado na avaliação de estoques.

Alternativamente, tem sido utilizada metodologia baseada em distribuições de freqüência de comprimento para determinação do crescimento, cuja maior virtude é de poder se basear em informações associadas a estatística pesqueira, o que reduz seu custo e permite maior sustentabilidade operacional (e.g.
RufFino \& IsAac 1995, Matheus \& Estupiñan 2002). Assim, é importante desenvolver tecnologia de análise a partir destas informações, calibrando a partir dos métodos baseados em estruturas rígidas.

O uso de parâmetros da dinâmica estimados no passado, para avaliação de populações em anos correntes é útil e muitas vezes é a única opção disponível, mas deve ser evitado por não considerar mudanças recentes na dinâmica da espécie causadas pela própria pesca ou por mudanças ambientais. Alternativamente, é importante também utilizar metodologia que permita uso de dados atualizados nos modelos, o que é viabilizado por métodos que utilizam distribuições de freqüências de comprimento.

Comparando com os parâmetros de crescimento estimados para o Baixo Rio Negro, seus afluentes e no Baixo Solimões (BARThem \& Fabré 2004), vimos que os valores de $\mathrm{k}$ estimados entre 0,54-0,68 $\mathrm{ano}^{-1}$, estão dentro da amplitude dos valores obtidos no presente trabalho $\left(0,43-0,72\right.$ ano $\left.^{-1}\right)$. Esta similaridade fortalece a percepção de estabilidade na condição do estoque explotado e confirma a tendência de alto crescimento para espécie, como tem sido registrado para outros caraciformes migradores da Amazônia, como S. taeniurus, o qual apresentou k de 0,50 ano ${ }^{-1}$ e $P$. nigricans, com k de 0,460,50 ano $^{-1}$ (BARThem \& Fabré 2004). Por outro lado, a elevada taxa de crescimento estimada para o Madeira $\left(0,72\right.$ ano $\left.^{-1}\right)$ não é típica e pode ser devida a mecanismos compensatórios de parte da população, tais como apresentados por Rochet (1998) ou Mangel \& Munch (2005). A elevada explotação no Madeira 

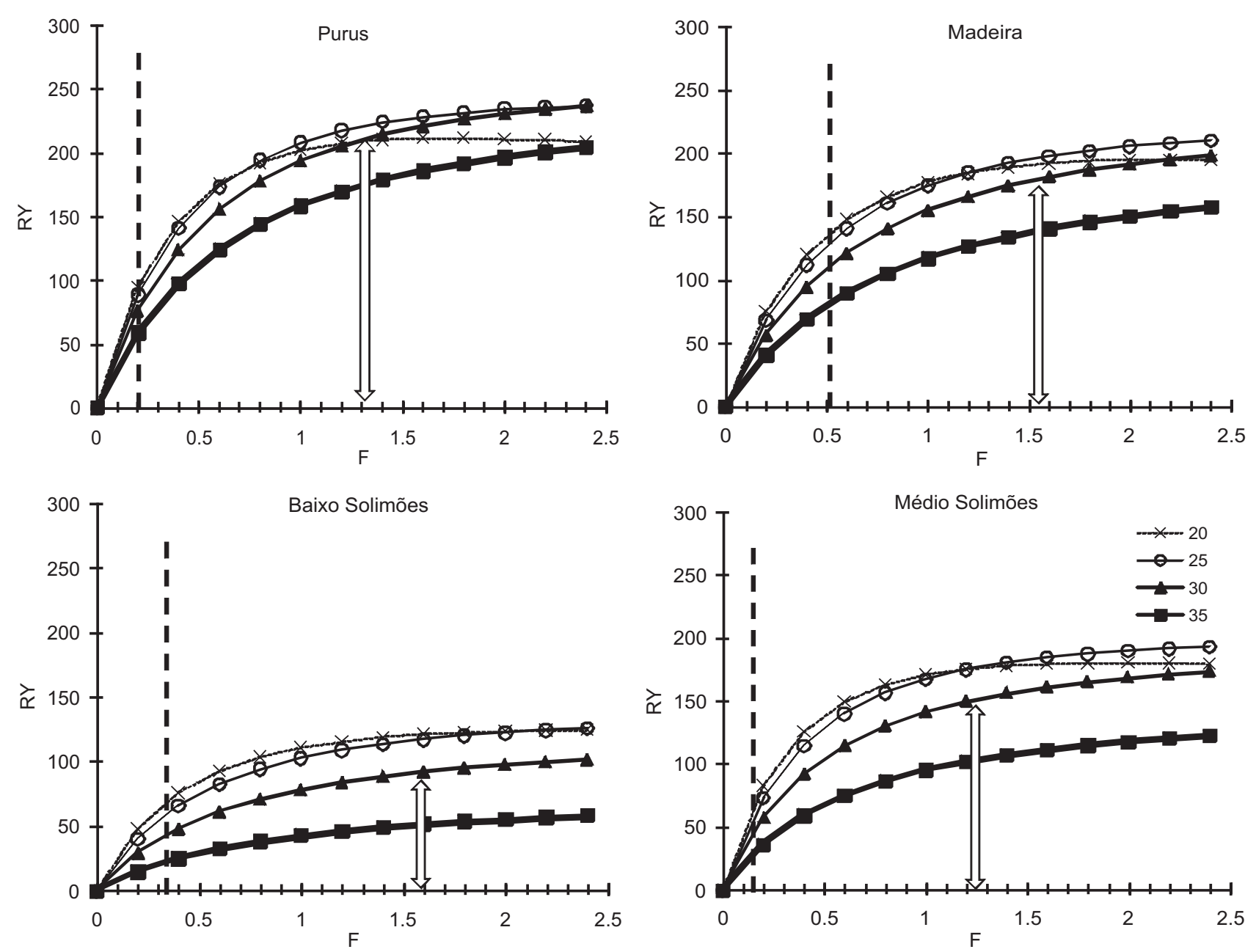

Figura 8. Curvas de rendimento por recruta $(Y / R)$ da matrinxã por sub-sistema contra a mortalidade por pesca ( $F)$ para diferentes comprimentos de recrutamento pesqueiro $(\mathrm{em} \mathrm{cm}$ ) indicados na legenda. A linha tracejada vertical indica o nível de $\mathrm{F}$ atual e a seta dupla vertical ao nível estimado de $\mathrm{F}_{0,1}$.

já foi descrita por Goulding (1979) na década de 1970, a qual pode ter ativado tais mecanismos compensatórios devido à diminuição do estoque de $B$. amazonicus neste rio.

Os comprimentos assintóticos $\left(\mathrm{L}_{\infty}\right)$ publicados em BARTHEM \& Fabré (2004) de 41-45 cm de comprimento padrão para a espécie, mostram-se semelhantes aos estimado neste trabalho (43-51 cm CF). Exceto entre o Purus e o baixo Solimões, nas análises comparativas entre sub-sistemas também houveram diferenças notórias dos parâmetros de crescimento (25-67\%), mortalidade total (13-66\%) e natural (16-37\%), sendo indício de unidades populacionais diferenciadas. Como as diferenças entre o Purus e o baixo Solimões foram das menores (5\% para o $\mathrm{k}, 16 \%$ para $\mathrm{Z}$ e $0,09 \%$ para $\mathrm{M}$ ), e a proximidade entre eles, os recursos explotados nestes sistemas provavelmente devem ser afins em termos populacionais e pesqueiros.
O valor do índice de performance de crescimento $\left(\varnothing^{\prime}\right)$ ficou em torno de três para todos os sub-sistemas avaliados, o que sugere uma satisfatória (menor do que cinco) estimativa dos parâmetros de crescimento, uma vez que para uma mesma espécie este índice não deve variar muito.

As curvas de crescimento da espécie mostram semelhanças entre as potenciais unidades populacionais avaliadas no rio Solimões (Baixo e Médio), mas as diferenças destas com as curvas do Purus e Madeira são mais intensas. Um problema no uso da metodologia do ELEFAN/FISAT é a falta de informação da dispersão dos dados no ajuste do modelo (RosemberG \& BEDDINGTON 1987), o que não permite testes estatísticos comparando as curvas. Entretanto, as curvas desenhadas facilita ao leitor efetuar a análise comparativa, permitindo a discussão com pesquisadores e gestores a partir das curvas impressas. 
Como resultado, sugerimos que três unidades populacionais devam ser testadas por outros métodos, tais como marcaçãorecaptura e genéticos, sendo formadas pelos sub-sistemas: 1) Purus, 2) Madeira e 3) Solimões (Baixo e Médio).

Comparando as taxas de mortalidade natural, estimadas por BATisTa (2001) entre 1,2-1,60 ano $^{-1}$ e entre 1,06-1,76 neste trabalho, temos indícios de estabilidade de médio prazo ( \pm 10 anos) na mortalidade natural e valores mais confiáveis para uso em modelos analíticos.

Os resultados obtidos para a taxa de explotação pesqueira para os sub-sistemas estudados estão sujeitos as incertezas no valor de $\mathrm{M}$, já que $\mathrm{F}$ é obtido usando $\mathrm{M}$, porém como o método é padronizado entre sub-sistemas, a comparação entre estes está menos sujeita a vícios. Assim, a menor taxa de explotação registrada para o Purus, particularmente em relação à observada nos sub-sistemas Madeira e Baixo Solimões, é bastante interessante por aquele sub-sistema apresentar a maior captura da espécie na região em estudo. Se observamos que tanto Z quanto $\mathrm{M}$ são mais elevadas no Madeira, mas ambas são mais similares no Purus e Baixo Solimões, temos indícios de que as condições de sobrevivência da espécie no Madeira devem ser mais críticas, ao contrário do que ocorre no Purus. A elevada integridade biótica do sub-sistema Purus e do Médio Solimões podem ser razão para terem apresentado os menores valores de M. Por outro lado, a condição impactada do rio Madeira (BARTHEM et al. 2004), associada a existência de mercados locais e sub-regionais (e.g. Porto Velho, Humaitá e Manicoré) (CARdoso \& Freitas 2007) pode estar indicando a necessidade de cuidados especiais com a gestão deste recurso neste sub-sistema.

A análise comparativa de curvas de rendimento por recruta com diferentes valores para $\mathrm{M}$ é importante devido as dificuldades inerentes á obtenção de estimativas realistas deste coeficiente (Sparre \& Venema 1997), permitindo avaliar os efeitos da variação deste coeficiente.

O rendimento por recruta demonstrou diferenças entre os sub-sistemas estudados, indicando que o sub-sistema Madeira estaria mais próximo de atingir o máximo rendimento por recruta em contraste aos demais. Avaliações de estoques pesqueiros no neotrópico têm apresentado resultados diversificados. No Pantanal, Mateus \& Estupiñán (2002) verificaram que o rendimento por recruta máximo da piraputanga Brycon hilarii (Valenciennes, 1850) comercializada no mercado do porto de Cuiabá ainda não foi alcançado, concluindo que seu estoque não estava sobre-explotado. Similar conclusão foi obtida por Mateus \& Petrere (2004) sobre o pintado Pseudoplatystoma corruscans (Spix \& Agassiz, 1829) no pantanal matogrossense. Na Amazônia, Petrere (1983) não registrou sobrepesca do tambaqui Colossoma macropomum (Cuvier, 1816) com dados dos anos 1970 na Amazônia Central, porém Ruffino \& IsAAC (1995) encontraram sobrepesca de crescimento no baixo-Amazonas nos anos 1990. Já BAtista (2001), avaliando o nível de explotação de matrinxã na Amazônia Central com dados de
1994 a 1996 não detectou sobre-explotação, assumindo existir apenas uma unidade populacional. Porém, os parâmetros populacionais estimados no presente trabalho permitem com que sigamos considerando a possibilidade da existência de três unidades populacionais: Purus, Madeira e Solimões (baixo e médio), justificando uma avaliação por unidade geográfica diferenciada. Os casos no Pantanal, referentes a B. microlepis (Valenciennes, 1850), P. corruscans e Piaractus mesopotamicus (Holmberg, 1887) são mais provavelmente uma única unidade populacional, mas na mega-bacia Amazônica há distâncias muito mais amplas e condições hidro-ecológicas muito complexas (SIOLI 1984, JunK 1997), que permitem a formação de populações diferenciadas, o que já foi sugerido mesmo para a espécie grande migradora dourada, Brachyplatystoma rousseauxii (Castelnau, 1855), e que pode estar ocorrendo para B. amazonicus, devendo ser tema prioritário de investigação para as principais espécies explotadas.

Sejam confirmadas como unidades populacionais separadas ou não, os resultados indicam que a espécie ainda se encontra sub-explotada para geração de rendimentos máximos. Porém, considerando a insipiência do sistema de controle da pesca por parte dos gestores e as deficiências no monitoramento da atividade e dos recursos, recomenda-se que as atuais taxas de explotação pesqueira (F/Z) sejam mantidas. Mais estudos comparativos por sub-sistemas, tanto genéticos quanto de crescimento utilizando estruturas rígidas, são necessários para que se possa fazer comparações estatísticas, permitindo inferir com mais segurança sobre aumento ou redução do esforço em cada sub-sistema, além de definir as unidades populacionais mais seguramente. Também deve-se ter clareza na definição dos objetivos do uso da espécie e que rendimentos máximos não são compatíveis com uma pesca de subsistência importante, a qual requer maiores densidades de peixes no ambiente para garantia da segurança alimentar da família (CADDY \& MAHON 1996) ou mesmo com os propósitos sociais de manutenção da renda estável em uma pesca artesanal com tantos pescadores e famílias envolvidas (BATISTA et al. 2004). Devemos sim considerar que estes resultados são pontos referenciais (CADDY \& MAHON 1996) a serem considerados na definição do manejo da pesca da espécie.

\section{AGRADECIMENTOS}

Agradecemos ao ProVárzea/Ibama, Conselho Nacional de Desenvolvimento Científico e Tecnológico, Fundação de Amparo a Pesquisa do Estado do Amazonas, Fundo Nacional do Meio Ambiente e Universidade Federal do Amazonas, pelo apoio material ou financeiro na execução deste trabalho e à Colônia de Pescadores de Manaus e aos pescadores que com suas informações, tornaram possível este trabalho. Também somos gratos aos técnicos que colaboraram no desenvolvimento das atividades, particularmente a L.M.F. Amazonas, L.F.S. Amazonas e T.M.P. Braga e aos demais pesquisadores do Programa Integrado de Recursos Aquáticos e da Várzea-PYRÁ. 


\section{LITERATURA CITADA}

Barthem, R.B. \& N.N. Fabré. 2004. Biologia e diversidade dos recursos pesqueiros na Amazônia, p. 17-62. In: M.L. Ruffino (Ed.). A pesca e os recursos pesqueiros na Amazônia. Manaus, Ibama, 272p.

Barthem, R.B. \& M. Petrere JR. 1996. Fisheries and population dynamics of Brachyplatystoma vaillantii (Pimelodidae) in the Amazon Estuary, p. 329-340. In: R.M. Meyer; C. Zhang; M.L. Windsor,; B.J. McCaY,; L.J. Hushak \& R.M. Muth (Eds). Fisheries resource utilization and policy. Proceedings of the World Fisheries Congress, Theme 2. New Delhi, Oxford \& IBH Publishing Co., 535p.

Barthem, R.B.; P. Charvet-Almeida; L.F.A. Montag \& A.E. Lanna. 2004. Amazon Basin, GIWA Regional assessment 40b. Sweden, University of Kalmar/UNEP, 60p.

BATISTA, V.S. 2001. Biologia e administração pesqueira de alguns caraciformes explotados na Amazônia Central. Manaus, UFAM, 131p.

Batista, V.S. \& M. Petrere Jr. 2003. Characterization of the commercial fish production landed at Manaus, Amazonas State, Brazil. Acta Amazônica 33 (1): 53-66.

Batista, V.S.; C.E.C. Freitas; A.J. Inhamuns \& D. Freire-Brasil. 2000. The Fishing Activity of the River People in the Floodplain of the Central Amazon, p. 417-432. In: W.J. Junk; J.J. Ohly, M.T.F. Piedade \& M.G.M. Soares (Eds). The central Amazon floodplain actual use and options for a sustainable management. Leiden, Backhuys Publishers, 584p.

Batista, V.S.; V.J. IsAaC \& J.P Viana. 2004. Capítulo 2 - Exploração e manejo dos recursos pesqueiros da Amazônia, p. 57135. In: M.L. Rufrino (Ed.). A pesca e os recursos pesqueiros na Amazônia. Brasília, Ibama, 272p.

Beverton, R.J.H. \& S.J. Holt. 1956. A review of methods for estimating mortality rates in exploited fish populations, with special reference to sources of bias in catch sampling. Rapports et Procès-Verbaux des Réunions, Conseil International pour l' Exploration de la Mer 140: 67-83.

Beverton, R.J.H. \& S.J. Holt. 1957. On the dynamic of exploited fish populations. Fisheries Investments Series 2 (19): 533p.

Caddy, J.F. \& R. MAHON. 1996. Reference points for fisheries management. Roma, FAO Fisheries Technical Paper 347, 83p.

Cardoso, R.C. \& C.E.C. Freitas. 2007. Desembarque e esforço de pesca da frota pesqueira comercial de Manicoré (Médio Rio Madeira), Amazonas, Brasil. Acta Amazônica 37 (4): 605612.

Fabré, N.N. \& U. SaInt-Paul. 1998. Annulus formation on scales and seasonal growth of the Central amazonian anostomid Schizodon fasciatus. Journal of Fish Biology 53: 1-11.

Ferreira, E.J.G.; J. Zuanon \& G.M. Santos. 1996. Peixes Comerciais do Médio Amazonas: região de Santarém, Pará. Brasília, Edições Ibama, 214p.

Gayanilo Jr, F.C. \& D. Pauly. 1997. FAO-ICLARM Stock Assessment
Tools: Reference Manual. Roma, FAO Computerized Information Series/Fisheries 8, 262p.

Goulding, M. 1979. Ecologia da pesca no Rio Madeira. Manaus, CNPq/INPA, 172p.

Goulding, M. 1980. The fishes and the forest. Explorations in Amazonian natural history. Berkeley, University of California Press, 280p.

GulLAND, J.A. 1983. Fish stock assessment: a manual of basic methods. Roma, FAO/Wiley Series on Food and Agriculture, $223 p$.

Hilborn, R. \& C.J. Whaters. 1992. Quantitative fisheries stock assessment: choice, dynamics and uncertainty. New York, Chapman and Hall, 785p.

Iвама. 2003. Estatística da pesca 2001. Brasília, Ibama, 124p. IвAма. 2004a. Estatística da pesca 2002. Brasília, Ibama, 129p. Iвама. 2004b. Estatística da pesca 2003. Brasília, Ibama, 137p. IвАмA. 2005. Estatística da pesca 2004. Brasília, Ibama, 136p. Івама. 2007. Estatística da pesca 2005. Available online at: http://www.ibama.gov.br/rec_pesqueiros/download.php? id_download $=113$ [Accessed: 07/III/2008]

IsAaC, V.J. \& M.L. RufFINo. 1996. Population dynamics of tambaqui (Colossoma macropomum Cuvier 1818) in the lower Amazon, Brazil. Fisheries Management and Ecology 3: 315-333.

Junk, W.J. 1997 The Central Amazon Floodplain: ecology of a pulsing system. Berlin, Springer-Verlag, Ecological Studies $126,525 \mathrm{p}$.

Junk, W.J. \& H.J. Krambeck. 2000. Climate and hidrology, p. 95108, In: W.J. Junk; J.J. Ohly; M.T.F. Piedade \& M.G.M. Soares (Eds). The Central Amazon Floodplain: actual use and options for a sustainable management. Leiden, Backhuys Publishers, 584p.

KING, M. 1995. Fisheries biology, assessment and management. Oxford, Fishing News Books, Blackwell Science, 341p.

LimA, F.C.T. 2003. Characidae - Bryconinae (Characins, tetras), p. 174-181. In: R.E. Reis; S.O. Kullander \& C.J. Ferraris JR (Eds). Checklist of the freshwater fishes of South and Central America. Porto Alegre, EDIPUCRS, 742p.

Longhurst, A.R. \& D. PAUly. 1987. Ecology of tropical oceans. San Diego, Academic Press, 407p.

Mangel, M. \& S.B. Munch. 2005. A Life-History Perspective on Short- and Long-Term Consequences of Compensatory Growth. The American Naturalist 166: E155-E176.

Mateus, L.A.F \& G.M.B. Estupiñ́n. 2002. Fish stock assessment of piraputanga Brycon microlepis in the Cuiabá river basin, Pantanal of Mato Grosso. Brazilian Journal of Biology 62 (1): 165-170.

Mateus, L.A.F. \& M. Petrere Jr. 2004. Age, growth and yield per recruit analysis of the pintado, Pseudoplatystoma corruscans (Agassiz, 1829) in the Cuiabá River basin, Pantanal Matogrossense, Brazil. Brazilian Journal of Biology 64 (2): 257264.

PAULY, D. 1983. Algunos métodos simples para la evaluación de recursos pesqueros tropicales. Roma, FAO Documen- 
to Técnico de Pesca 234, 49p.

PAULY, D. 1984. Fish population dynamics in tropical waters: a manual for use with programmable calculator. Manila, ICLARM Studies and Reviews, 325p.

Pauly, D \& G.R. Morgan. 1987. Length-based methods in fisheries research. Manila, ICLARM 13, Conference Proceedings, 486p.

Pauly, D. \& J.L. Munro. 1984. Once more on the comparison of growth in fish and invertebrates. Fishbyte 2 (1): 21.

Petrere Jr, M. 1978. Pesca e esforço de pesca no estado do Amazonas. I. Esforço e captura por unidade de esforço. Acta Amazônica 8: 439-454.

Petrere Jr, M. 1983. Yield per recruit of the Tambaqui, Colossoma macropomum Cuvier, in the Amazonas States, Brazil. Journal of Fish Biology 22: 133-144.

Rochet, M.J. 1998. Short-term effects of fishing on life history traits of fishes. ICES Journal of Marine Science 55: 371-391.

Rosenberg, A.A. \& J.R. Beddington. 1987. Monte-Carlo testing of two methods for estimating growth from length-frequency data with general conditions for their applicability, p. 283298. In: D. Pauly \&. G.R. Morgan (Eds). Length-based methods in fisheries research. Manila, ICLARM 13, Conference Proceedings, 486p.

RufFINO, M.L. \& V.J. ISAAC. 1995. Life cycle and biological parameters of several Brazilian Amazon fish species. ICLARM Quartely 18 (4): 41-45.

Submitted: 18.X.2007; Accepted: 09.III.2009.

Editorial responsibility: Paulo de Tarso da Cunha Chaves
Saint-Paul, U. \& P.B. Bayley. 1979. A situação da pesca na Amazônia Central. Acta Amazônica 9 (Supl.): 109-114.

Santos, G.M.; E.J.G. Ferreira \& J.A.S. Zuanon. 2006. Peixes comerciais de Manaus. Manaus, Ibama-AM/ProVárzea, $144 \mathrm{p}$.

Santos-Filho, L.C. \& V.S. Batista. 2005. Determinação de idade e crescimento do mapará (Hypophthalmus marginatus) na Amazônia Central. Acta Amazônica 35 (1): 85-92.

Sioli, H. The Amazon: limnology and landscape ecology of a mighty tropical river and its basin. Dordrecht, W. Junk Publications, 763p.

SMith, M. 1979. A pesca no Rio Amazonas. Manaus, CNPq/ INPA, 154p.

SокAL, R.R.\& F.J. Rohlf. 1995. Biometry. San Francisco, W.E. Freeman, $3^{\text {th }}$ ed, $887 \mathrm{p}$.

SPARRe, P. \& S.C. Venema. 1997. Introdução à avaliação de mananciais de peixes tropicais. Parte 1: Manual. Roma, FAO Documento técnico sobre as pescas 306/1, Rev. 2, 404p. Sudepe. 1988. Diagnóstico do setor pesqueiro do Estado do Amazonas. Manaus, SUDEPE, 15p.

von Bertalanffy, L. 1938. A quantitative theory of organic growth. Human Biology 10: 181-213.

Zaniboni-Filho, E. \& E.K. Resende. 1988, Anatomia das gônadas, escala de maturidade e tipo de desova do matrinxã, Brycon cephalus (Günther, 1869) (Teleostei: Characidae). Revista Brasileira de Biologia 48 (4): 833-844. 